\title{
Tick-Borne-Associated Illnesses in the Pediatric Intensive Care Unit
}

\author{
Kaila Lessner ${ }^{1}$ Conrad Krawiec $^{2}$ \\ ${ }^{1}$ Department of Pediatrics, Eastern Virginia Medical School, Norfolk, \\ Virginia, United States \\ 2 Department of Pediatrics, Penn State Children's Hospital, Pediatric \\ Critical Care Medicine, Hershey, Pennsylvania, United States \\ J Pediatr Infect Dis 2020;15:269-275.
}

Address for correspondence Kaila Lessner, MD, Department of Pediatrcis, Eastern Virginia Medical School, 825 Fairfax Avenue Suite 310, Norfolk, VA 23507, United States (e-mail: LessneKM@evms.edu).
Abstract
Keywords
- Rocky Mountain- spotted fever
- Lyme disease
- human ehrlichiosis and anaplasmosis
- babesiosis

When unrecognized and antibiotic delay occurs, Lyme disease, Rocky Mountainspotted fever, babesiosis, and human ehrlichiosis and anaplasmosis can result in multiorgan system dysfunction and potentially death. This review focuses on the early recognition, evaluation, and stabilization of the rare life-threatening sequelae seen in tick-borne illnesses that require admission in the pediatric intensive care unit.

\section{Introduction}

In the United States, the reported number of tick-borne diseases each year is increasing. ${ }^{1}$ This is at least partly due to the rising average temperatures caused by global warming which is elongating the season of tick activity and enhances their survival in the environment. ${ }^{2}$ Studies have shown that increased temperatures influence the life cycle of ticks, as well as the ecosystem overall, creating an environment more prone to the spread of tick borne illnesses., ${ }^{3,4}$ Because of this, tick-borne illnesses have now begun to appear in the fall, winter, and spring months, in addition to the summer due to changes in the epidemiology of these diseases from rising temperatures. ${ }^{5}$ Environmental conditions that are suitable for tick survival (i.e., persistence of shrubs), increased tick activity, and a reported lack of recognition of the occurrence of a tick bite place children at risk of exposure. . $^{2,3,6,7}$

The Centers of Disease Control and Prevention (CDC) recognizes the threat of many diseases worldwide, including old diseases which have evolved, mutated, and gained new features. ${ }^{2}$ If unrecognized, these tick-borne-associated illnesses ( - Table 1 ) can have life-threatening sequalae. ${ }^{8}$ Clinicians must quickly identify and treat the tick-borne illness and any complications to ensure the best possible outcome. This article focuses on the most common tick-borne illnesses, their rare life-threatening sequelae, and therapeutic options that allow prompt stabilization.

\section{Tick-Borne Illnesses}

\section{Lyme Disease}

\section{Key Points}

- The clinical manifestations of Lyme disease vary depending on the stage of illness, thus a careful history and objective clinical findings is required to identify this condition.

- When a pediatric intensivist is faced with a patient with carditis, heart block, or myopericarditis, early Lyme disease should be considered.

- Diagnose via a two-tier method with enzyme-linked immunosorbent assay (ELISA) and immunoglobulin (Ig)$\mathrm{M}$ and IgG immunoblots.

- For neurologic or cardiac sequalae, intravenous ceftriaxone, cefotaxime, or penicillin should be administered. received

June 16, 2020

accepted after revision

September 1, 2020

published online

October 15, 2020
Copyright @ 2020 by Georg Thieme

Verlag KG, Stuttgart · New York
DOI https://doi.org/

10.1055/s-0040-1717149. ISSN 1305-7707. 
- Because of an increased risk of coinfection, empiric therapy with doxycycline should be administered in endemic areas.

\section{Brief Background}

Lyme disease is commonly seen in the northeast and midAtlantic regions. ${ }^{9,10}$ Expansion of this disease has been recently noted in the Midwest. ${ }^{9,11}$ It is transmitted via an Ixodes tick bite infected with Borrelia burgdorferi with an average incubation period of approximately 2 to 3 weeks. $^{12,13}$

\section{Life-Threatening Clinical Presentation}

Lyme disease develops in three stages. First, the typical erythema chronicum migrans rash and nonspecific symptoms develop, followed by wide hematogenous dissemination resulting in preferential involvement of specific organs, leading to joint pain, neurological complications, Borrelia lymphocytoma or Lyme carditis. ${ }^{11,14-16}$ A late manifestation of this disease includes arthritis and acrodermatitis chronica atrophicans. $^{15}$

Patients with early Lyme disease may develop Lyme carditis, leading to atrioventricular (AV) heart block, myopericarditis or first, second, or third degree heart block. ${ }^{15}$ Heart block most commonly occurs above the bundle of His, including the AV node. Symptoms include syncope, dyspnea, or chest pain. ${ }^{16}$

Patients with Lyme meningitis are less likely to be febrile but are often sicker for a longer duration than those with viral meningitis. These patients often have erythema migrans, cranial nerve palsy, or papilledema. ${ }^{16}$

\section{Diagnosis}

The diagnosis of early localized Lyme disease can be made clinically (presence of characteristic erythema migrans lesion in a patient who resides in an endemic area). ${ }^{17}$ All other manifestations of Lyme disease require serologic testing. ${ }^{18}$ Testing is typically two-tiered, involving a polyvalent ELISA then IgM and IgG immunoblots. ${ }^{14,16}$ A positive or equivocal ELISA with a positive IgM Western immunoblot ( $\geq 2$ of 3 bands) and positive IgG result ( $\geq 5$ of 10 bands) has a sensitivity of approximately 70 to $100 \%$ and a specificity of $>95 \%$ for disseminated Lyme disease. ${ }^{18}$

Lumbar puncture is indicated for patients with a strong clinical suspicion of cerebral nervous system involvement. This may have a lower negative predictive value and the cerebrospinal fluid (CSF) results may be difficult to differentiate from aseptic meningitis. ${ }^{14,16,19}$ A high index of suspicion for Lyme meningitis should occur if the patient is older (greater than 10 years of age), has a CSF specimen with a predominance of mononuclear cells (fewer white blood cells), longer duration of symptoms (more than 12 days), presence of cranial nerve palsy, and presence of papilledema. ${ }^{19-22}$

Echocardiogram and cardiac magnetic resonance imaging (MRI) can determine if there is pericardial involvement or left ventricular dysfunction. ${ }^{15}$ The presence of transmural inflammatory infiltrates with characteristic band-like endo- cardial lymphocytic infiltration on endomyocardial biopsy is gold standard for diagnosing myocarditis. ${ }^{15}$

\section{Stabilization}

The first-line treatment is doxycycline (oral $2 \mathrm{mg} / \mathrm{kg} /$ dose twice daily for 10 to 21 days depending on clinical manifestation type). ${ }^{16}$ Amoxicillin or cefuroxime axetil are alternatives for erythema migrans for children under 8 years. Macrolides and first-generation cephalosporins should not

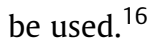

In cases of Lyme meningitis and carditis, intravenous ceftriaxone (50 to $75 \mathrm{mg} / \mathrm{kg} /$ dose once daily) is recommended. ${ }^{15,16}$ Alternatives include cefotaxime or penicillin. ${ }^{15,16}$

For symptoms of increased intracranial pressure, serial lumbar punctures, acetazolamide, or shunting may be required. $^{23}$

Patients with Lyme carditis should be hospitalized for monitoring due to possible decompensation and need for a temporary pacemaker. ${ }^{15}$ Due to a direct effect on the atrioventricular node rather than an indirect vagotonic effect, most patients do not respond to atropine administration. ${ }^{24}$

There is an increased risk of coinfection with other infections that can be transmitted by Ixodes ticks, particularly babesiosis. ${ }^{11,16}$ Empiric antibiotic therapy with doxycycline should be administered. ${ }^{11,16}$

\section{Rocky Mountain-Spotted Fever}

\section{Key Points}

- When an intensivist is faced with a patient with nonspecific flu-like symptoms and shock, Rocky Mountainspotted fever (RMSF) should be considered as the classic triad of fever, rash, and headache is not always initially present.

- The best diagnostic test are immunofluorescent antibody assays.

- Common laboratory abnormalities include thrombocytopenia and hyponatremia.

- Empiric treatment with doxycycline is recommended in suspected cases of RMSF to reduce the risk of mortality.

\section{Brief Background}

In the United States, cases of RMSF were reported with an incidence of 2 to 4 per million among 1- to 19-year-old children between 2000 and 2008 in the southeastern and southcentral region. ${ }^{25}$ Most cases occur between April and September and rates are increasing. ${ }^{25}$ After inoculation from a feeding tick (Dermacentor variabilis [the American dog tick], Dermacentor andersoni (the Rocky Mountain wood tick), or Rhipicephalus sanguineus (common brown dog tick)], the Rickettsia rickettsii bacteria infect the endothelial and vascular smooth muscle cells in the brain, skin, liver, lungs, kidneys, and gastrointestinal tract with patients becoming symptomatic 5 to 7 days after exposure. ${ }^{26-28}$ Many possible disease manifestations result from the small- to medium-sized vessel vasculitis produced. ${ }^{27}$ A delay of antibiotic treatment of more than 5 days can result in the mortality risk increasing more than three folds. ${ }^{8,29,30}$ 


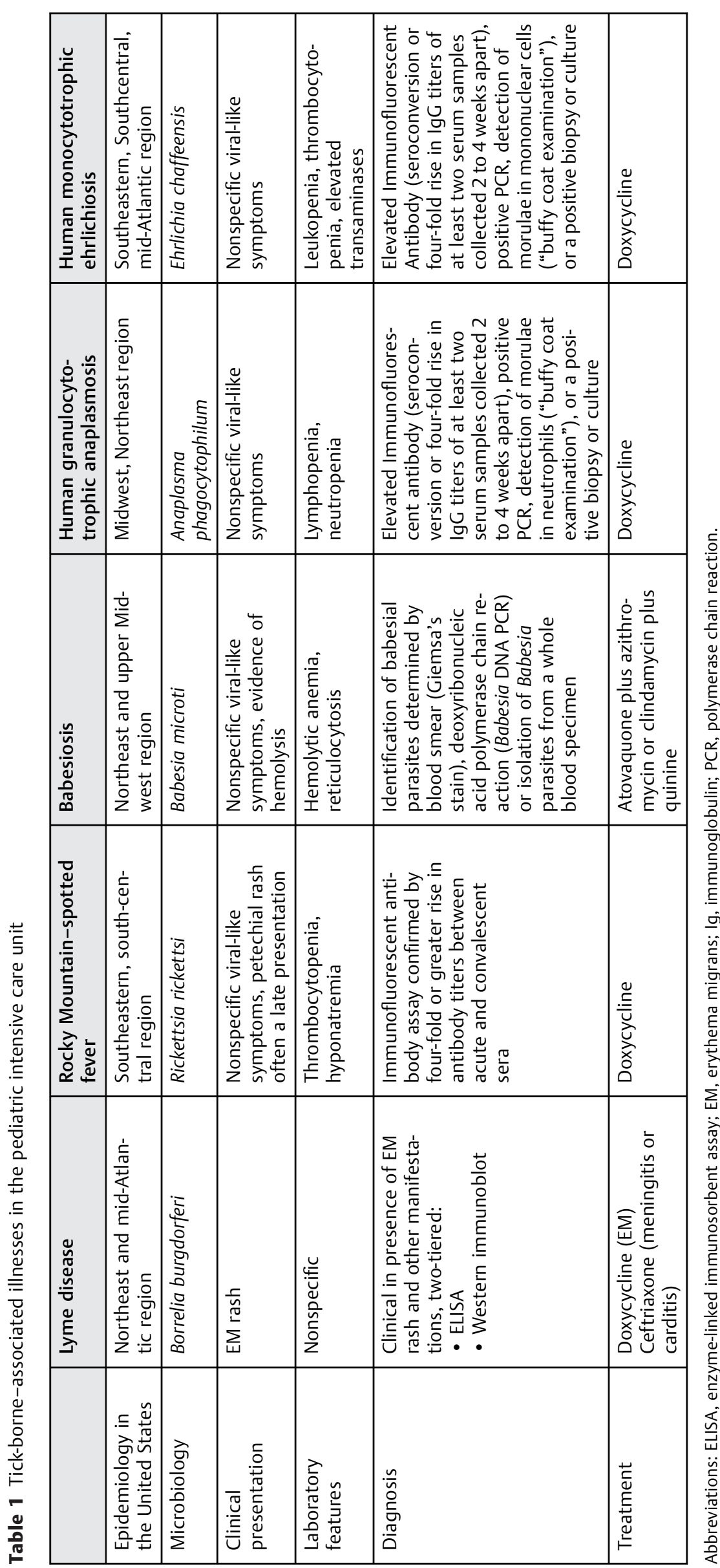




\section{Life-Threatening Clinical Presentation}

The classic triad of fever, rash, and headache is rarely present early in the course. ${ }^{8,25,31}$ Nonspecific symptoms, such as myalgia, abdominal pain, nausea, vomiting, and conjunctival injection can be mistaken for common viral or bacterial infections, leading to a delayed diagnosis. ${ }^{29,32}$ The rash may present as petechial on the ankles, progressing centrally or as a maculopapular rash. ${ }^{25}$ The intensivist should be aware that petechial rashes often manifest later in the disease course (day 5 or 6 ), may be difficult to distinguish from meningococcemia, and is a sign of severe disease. ${ }^{30}$ Thus, a high clinical suspicion is required (especially in an immunocompetent patient), and every attempt should be made to treat the patient before petechiae develop. ${ }^{33}$ The rash can, however, be completely absent. ${ }^{34}$

Critically ill patients can develop hypotension, shock, renal failure, seizure, stupor, coma, encephalitis, hemorrhagic manifestations, noncardiogenic pulmonary edema, or acute respiratory distress syndrome. ${ }^{35}$ When this occurs, they are five times more likely to succumb to this illness due to airway compromise, hypoxemia, and hemodynamic instability. ${ }^{35}$

\section{Diagnosis}

The gold standard for diagnosis is immunofluorescent antibody assays (IFA) confirmed by a four-fold or greater rise in serum antibody titers between acute (as IgM and IgG antibodies against Rickettsia are not detectable during the first 7 days of illness) and convalescent sera. ${ }^{25}$

Laboratory features include thrombocytopenia and hyponatremia. ${ }^{28}$ The white blood cell count is typically normal, with an increased number of immature bands. ${ }^{28}$ Other laboratory abnormalities include elevated transaminases, elevated blood urea nitrogen (BUN) level, and creatinine, hyperbilirubinemia, low-serum albumin, leukocytosis, and leukopenia. ${ }^{25}$ CSF studies often shows a lymphocytic pleocytosis $(<100$ cells $/ \mu \mathrm{L}){ }^{28}$

MRI findings in RMSF encephalitis may show a starry sky pattern on T1, T2, and diffusion-weighted imaging. ${ }^{27}$

\section{Stabilization}

Patients requiring intravascular volume support warrant hospital admission. ${ }^{25}$ If antimicrobial administration is delayed, multiorgan system dysfunction can occur. Thus, treatment with oral or intravenous doxycycline (2.2 $\mathrm{mg} / \mathrm{kg} /$ dose every 12 hours for minimum of 5-7 days and continue for at least 3 days after fever resolution and clinical improvement) should be initiated without waiting for confirmation. ${ }^{28}$ In this disease (and any severe presentation of suspected tick-borne diseases), the American Academy of Pediatrics supports the use doxycycline in all age groups for less than 21 days. $^{32}$ Dental staining is a minor concern but the risk is outweighed by the potential life-threatening sequalae. $^{32}$

Children with severe RMSF are at risk for multiorgan system dysfunction. Neurologically, the patient may develop acute encephalopathy due to cerebral edema, sepsis, or increased intracranial pressure. ${ }^{36}$ The clinician must be prepared to provide supportive care including airway, fluid, and electrolytes, and intracranial pressure monitoring. Children with severe RMSF can develop acute respiratory failure due to loss of airway, pulmonary edema, or acute respiratory distress syndrome. ${ }^{28}$ Thus, these patients often require invasive mechanical ventilation. If shock develops, inotropic and vasopressor support is routinely required. ${ }^{25}$ Clinicians should be aware of the risk of peripheral vasoconstriction resulting in skin necrosis, gangrene, and the risk of amputation that may be potentiated by vasopressor therapy. ${ }^{37}$ In some cases, renal insufficiency can develop, thus, the clinician should be prepared to provide hemodialysis. $^{25}$

\section{Babesiosis}

\section{Key Points}

- When an intensivist is faced with a patient with fever, viral-like illness, and evidence of hemolysis, babesiosis should be considered.

- High-grade parasitemia $(\geq 10 \%)$ indicates severe babesiosis.

- Laboratory abnormalities include hemolytic anemia with elevated reticulocyte count.

- Diagnose with identification of babesial parasites in Giemsa's stains or DNA polymerase chain reaction (PCR).

- Severe cases may require exchange transfusion and should be performed at centers with expertise in apheresis.

- Treat with atovaquone and azithromycin if mild or moderate; clindamycin and quinine in severe cases and when requiring exchange transfusion.

\section{Brief Background}

Babesiosis is caused by intraerythrocytic protozoa. ${ }^{38}$ The parasite causes proinflammatory cytokines that stimulate the production of nitric oxide, which results in erythrocytic cellular damage. ${ }^{38}$ It is endemic to parts of New England, New York, New Jersey, Minnesota, and Wisconsin. ${ }^{16}$ Babesiosis is transmitted by Ixodes scapularis ticks with an incubation period of 1 to 4 weeks. ${ }^{39,40}$ Because it shares a vector with other tick-borne diseases, it often occurs as a coinfection most commonly with Borrelia burgdorferi and Anaplasma phagocytophlium. ${ }^{28}$

\section{Life-Threatening Clinical Presentation}

Babesiosis can go undiagnosed in healthy patients. ${ }^{38}$ Patients experience a viral-like illness, with fever, chills, sweats, myalgia, arthralgia, anorexia, nausea, vomiting, fatigue, and dark urine. ${ }^{16}$

Severe babesiosis is indicated by high-grade parasitemia ( $\geq 10 \%$ ), significant hemolysis or renal, hepatic or pulmonary compromise. ${ }^{16}$ Patients may experience acute respiratory failure, disseminated intravascular coagulation, congestive heart failure, coma, and renal failure. ${ }^{16,41,42}$

There have been case reports of splenic rupture. ${ }^{43}$ Symptoms include left upper quadrant abdominal pain, hypotension, and anemia. ${ }^{43}$ 


\section{Diagnosis}

Diagnostic criteria includes the presence of viral-like symptoms and identification of babesial parasites. The presence of parasites can be determined by blood smear (Giemsa's stains), deoxyribonucleic acid PCR (Babesia DNA PCR) or isolation of Babesia parasites from a whole blood specimen. ${ }^{44}$

Laboratory abnormalities include hemolytic anemia with elevated reticulocyte count, thrombocytopenia, proteinuria and elevated liver enzyme, BUN, and creatinine levels. ${ }^{16}$

\section{Stabilization}

Initial therapy includes atovaquone $(20 \mathrm{mg} / \mathrm{kg}$ every 12 hours) plus azithromycin $(10 \mathrm{mg} / \mathrm{kg} /$ day on day 1 followed by $5 \mathrm{mg} / \mathrm{kg} /$ day from day 2) or intravenous clindamycin ( $7-10 \mathrm{mg} / \mathrm{kg}$ every 6 to 8 hours) plus quinine $(8 \mathrm{mg} / \mathrm{kg}$ in every 8 hours) for 7 to 10 days. ${ }^{14,16,45}$ Hematocrit and percentage of parasitized erythrocytes should be monitored daily until the level of parasitemia has decreased to $<5 \%$ of erythrocytes. ${ }^{16}$

According to the Infectious Disease Society of America Guidelines, exchange transfusion and quinine plus clindamycin should be used for all patients with severe babesiosis, defined as high grade parasitemia $(\geq 10 \%)$, significant hemolysis, or renal, hepatic, or pulmonary compromise. $^{14,45}$

The clinician must be prepared to provide supportive care for severe illness, such as antipyretics, vasopressors, blood transfusions, exchange transfusions, mechanical ventilation, or dialysis. ${ }^{38}$

\section{Human Ehrlichiosis and Anaplasmosis}

\section{Key Points}

- Human granulocytotrophic anaplasmosis (HGA) and human monocytotrophic ehrlichiosis (HME) typically manifest as a self-limiting, flu-like illness. The intensivist should be aware; however, that severe complications can occur especially in the immunocompromised for HGA.

- Neurological complications are often severe in HME.

- Diagnosis requires clinical symptoms and positive laboratory results.

- Treat with doxycycline.

\section{Brief Background}

HGA and HME are rickettsial infections. ${ }^{46,47}$ HGA infects the neutrophils while HME infects monocytes and macrophages. ${ }^{48}$ HGA is endemically found within the Midwest and northeastern areas and its principal tick vector is Ixodes scapularis with an incubation period of 5 to 14 days. ${ }^{28,46}$ HME is mainly in the southcentral, southeastern, and midAtlantic regions and its tick vector is the lone star tick (Ambylomma americanum) with an incubation period of 5 to 14 days. ${ }^{47,49}$ Most cases are diagnosed between April and September. ${ }^{32,46,47}$ While reportedly rare, their prevalence is likely underreported in children due to mild infections that do not require medical care. ${ }^{46}$

\section{Life-Threatening Clinical Presentation}

Clinical manifestations of HGA and HME are often nonspecific, including rash, fever, malaise, chills, headache, myalgias, and abdominal pain. ${ }^{34,46,47,50}$ Neurologic manifestations are most commonly seen with HME. ${ }^{47}$

Life-threatening clinical signs seen in HGA and HME include encephalopathy, seizures, respiratory failure, shock, cardiac failure, myocarditis, and acute renal failure. ${ }^{34,47}$

\section{Diagnosis}

Diagnosis requires presence clinical findings and any of the following diagnostic tests: an elevated IFA (seroconversion or four-fold rise in IgG titers of at least two serum samples collected 2-4 weeks apart), positive PCR, detection of morulae in neutrophils (HGA) or mononuclear cells (HME; "buffy coat examination"), or a positive biopsy or culture. ${ }^{46,47,51}$ Laboratory abnormalities include thrombocytopenia, leukopenia, increased liver enzymes, elevated C-reactive protein, and hyponatremia. ${ }^{16,46,47,52}$

\section{Stabilization}

Doxycycline $(2.2 \mathrm{mg} / \mathrm{kg} /$ dose every 12 hours for $7-14$ days $)$ is the treatment of choice for both conditions. ${ }^{28}$ If contraindicated, rifampin $(10 \mathrm{mg} / \mathrm{kg} /$ dose twice per day $7-10$ days) may be used. ${ }^{16}$

HGA can have significant morbidity and mortality, especially in immunocompromised patients or cases of delayed treatment. ${ }^{16,52}$ The clinician should be prepared to provide neurologic, airway, respiratory, and hemodynamic support.

\section{Conclusion}

Tick-borne illnesses are associated with rare but life-threatening sequalae. Many cases present initially with unspecific, viral-like symptoms, and no known history of a tick bite. Early recognition, stabilization, and prompt referral to a tertiary center is recommended to reduce the risk of morbidity and mortality in pediatric patients who acquire these diseases.

\section{Funding}

None.

Conflict of Interest

None declared.

\section{References}

1 Center for Disease Control and Prevention. Lyme and other tickborne diseases increasing. Available at: https://www.cdc.gov/media/dpk/diseases-and-conditions/lyme-disease/index. html. Accessed February 1, 2020

2 El-Sayed A, Kamel M. Climatic changes and their role in emergence and re-emergence of diseases. Environ Sci Pollut Res Int 2020;27(18):22336-22352

3 Gray JS. Ixodes ricinus seasonal activity: Implications of global warming indicated by revisiting tick and weather data. Int J Med Microbiol 2008;298:19-24

4 Buczek AM, Buczek W, Buczek A, Bartosik K. The potential role of migratory birds in the rapid spread of ticks and tick-borne 
pathogens in the changing climatic and environmental conditions in Europe. Int J Environ Res Public Health 2020;17(06):17

5 Danielová V, Kliegrová S, Daniel M, Benes C. Influence of climate warming on tickborne encephalitis expansion to higher altitudes over the last decade (1997-2006) in the Highland Region (Czech Republic). Cent Eur J Public Health 2008;16(01):4-11

6 Beaujean DJMA, Gassner F, Wong A, Steenbergen JE, Crutzen R, Ruwaard D. Education on tick bite and Lyme borreliosis prevention, aimed at schoolchildren in the Netherlands: comparing the effects of an online educational video game versus a leaflet or no intervention. BMC Public Health 2016;16(01):1163

7 Nigrovic LE, Neville DN, Balamuth F, Bennett JE, Levas MN, Garro AC; for Pedi Lyme Net. A minority of children diagnosed with Lyme disease recall a preceding tick bite. Ticks Tick Borne Dis 2019;10(03):694-696

8 Mukkada S, Buckingham SC. Recognition of and prompt treatment for tick-borne infections in children. Infect Dis Clin North Am 2015;29(03):539-555

9 Beach CM, Hart SA, Nowalk A, Feingold B, Kurland K, Arora G. Increasing burden of Lyme carditis in United States children's hospitals. Pediatr Cardiol 2020;41(02):258-264

10 Bacon RM, Kugeler KJ, Mead PS. Centers for Disease Control and Prevention (CDC). Surveillance for Lyme disease-United States, 1992-2006. MMWR Surveill Summ 2008;57(10):1-9

11 Handel AS, Hellman H, Hymes SR. Two neonates with postnatally acquired tickborne infections. Pediatrics 2019;144(06): e20191937

12 Kobayashi T, Higgins Y, Samuels R, et al. Misdiagnosis of Lyme disease with unnecessary antimicrobial treatment characterizes patients referred to an academic infectious diseases clinic. Open Forum Infect Dis 2019;6(07):ofz299

13 Müllegger RR, Glatz M. Skin manifestations of Lyme borreliosis: diagnosis and management. Am J Clin Dermatol 2008;9(06): 355-368

14 Sanchez E, Vannier E, Wormser GP, Hu LT. Diagnosis, treatment, and prevention of lyme disease, human granulocytic anaplasmosis, and babesiosis: a review. JAMA 2016;315(16):1767-1777

15 Scheffold N, Herkommer B, Kandolf R, May AE. Lyme carditisdiagnosis, treatment and prognosis. Dtsch Arztebl Int 2015;112 (12):202-208

16 Wormser GP, Dattwyler RJ, Shapiro ED, et al. The clinical assessment, treatment, and prevention of Lyme disease, human granulocytic anaplasmosis, and babesiosis: clinical practice guidelines by the Infectious Diseases Society of America. Clin Infect Dis 2006; 43(09):1089-1134

17 Bratton RL, Whiteside JW, Hovan MJ, Engle RL, Edwards FD. Diagnosis and treatment of Lyme disease. Mayo Clin Proc 2008; 83(05):566-571

18 Moore A, Nelson C, Molins C, Mead P, Schriefer M. Current guidelines, common clinical pitfalls, and future directions for laboratory diagnosis of Lyme disease, United States. Emerg Infect Dis 2016;22(07):1169-1177

19 Shah SS, Zaoutis TE, Turnquist J, Hodinka RL, Coffin SE. Early differentiation of Lyme from enteroviral meningitis. Pediatr Infect Dis J 2005;24(06):542-545

20 Avery RA, Frank G, Glutting JJ, Eppes SC. Prediction of Lyme meningitis in children from a Lyme disease-endemic region: a logistic-regression model using history, physical, and laboratory findings. Pediatrics 2006;117(01):e1-e7

21 Garro AC, Rutman M, Simonsen K, Jaeger JL, Chapin K, Lockhart G. Prospective validation of a clinical prediction model for Lyme meningitis in children. Pediatrics 2009;123(05):e829-e834

22 Eppes SC, Nelson DK, Lewis LL, Klein JD. Characterization of Lyme meningitis and comparison with viral meningitis in children Pediatrics 1999;103(5 Pt 1):957-960

23 Kan L, Sood SK, Maytal J. Pseudotumor cerebri in Lyme disease: a case report and literature review. Pediatr Neurol 1998;18(05): 439-441
24 Pinto DS. Cardiac manifestations of Lyme disease. Med Clin North Am 2002;86(02):285-296

25 Woods CR. Rocky Mountain spotted fever in children. Pediatr Clin North Am 2013;60(02):455-470

26 Rydkina E, Sahni A, Baggs RB, Silverman DJ, Sahni SK. Infection of human endothelial cells with spotted Fever group rickettsiae stimulates cyclooxygenase 2 expression and release of vasoactive prostaglandins. Infect Immun 2006;74(09):5067-5074

27 Bradshaw MJ, Lalor KB, Vu N, Pruthi S, Bloch KC. Child neurology: Rocky Mountain spotted fever encephalitis. Neurology 2017;88 (11):e92-e95

28 Biggs HM, Behravesh CB, Bradley KK, et al. Diagnosis and management of tickborne rickettsial diseases: Rocky Mountain spotted fever and other spotted fever group rickettsioses, ehrlichioses, and anaplasmosis - United States. MMWR Recomm Rep 2016;65 (02):1-44

29 Traeger MS, Regan JJ, Humpherys D, et al. Rocky mountain spotted fever characterization and comparison to similar illnesses in a highly endemic area-Arizona, 2002-2011. Clin Infect Dis 2015;60 (11):1650-1658

30 Regan JJ, Traeger MS, Humpherys D, et al. Risk factors for fatal outcome from rocky mountain spotted Fever in a highly endemic area-Arizona, 2002-2011. Clin Infect Dis 2015;60(11): 1659-1666

31 Buckingham SC, Marshall GS, Schutze GE, et al; Tick-borne Infections in Children Study Group. Clinical and laboratory features, hospital course, and outcome of Rocky Mountain spotted fever in children. J Pediatr 2007;150(02):180-184

32 Buckingham SC. Tick-borne diseases of the USA: ten things clinicians should know. J Infect 2015;71(Suppl 1):S88-S96

33 Razzaq S, Schutze GE. Rocky Mountain spotted fever: a physician's challenge. Pediatr Rev 2005;26(04):125-130

34 Chapman AS, Bakken JS, Folk SM, et al; Tickborne Rickettsial Diseases Working Group CDC. Diagnosis and management of tickborne rickettsial diseases: Rocky Mountain spotted fever, ehrlichioses, and anaplasmosis-United States: a practical guide for physicians and other health-care and public health professionals. MMWR Recomm Rep 2006;55(RR-4):1-27

35 de Oliveira SV, Willemann MCA, Gazeta GS, Angerami RN, GurgelGonçalves R. Predictive factors for fatal tick-borne spotted fever in Brazil. Zoonoses Public Health 2017;64(07):e44-e50

36 Ravish ME, Krowchuk DP, Zapadka M, Shetty AK. A 6-year-old girl with fever, rash, and increased intracranial pressure. J Emerg Med 2013;45(02):186-189

37 Kirkland KB, Marcom PK, Sexton DJ, Dumler JS, Walker DH. Rocky Mountain spotted fever complicated by gangrene: report of six cases and review. Clin Infect Dis 1993;16(05):629-634

38 Paparone P, Paparone PW. Variable clinical presentations of babesiosis. Nurse Pract 2018;43(10):48-54

39 Gray JS, Estrada-Peña A, Zintl A. Vectors of babesiosis. Annu Rev Entomol 2019;64:149-165

40 Vannier EG, Diuk-Wasser MA, Ben Mamoun C, Krause PJ. Babesiosis. Infect Dis Clin North Am 2015;29(02):357-370

41 Blum S, Gattringer R, Haschke E, et al. The case: hemolysis and acute renal failure. Babesiosis. Kidney Int 2011;80(06):681-683

42 Hajicharalambous C, Rattu M, Leuchten S. A walk in the park: a case of babesiosis in the South Bronx. Clin Pract Cases Emerg Med 2018;2(01):61-63

43 Li S, Goyal B, Cooper JD, Abdelbaki A, Gupta N, Kumar Y. Splenic rupture from babesiosis, an emerging concern? A systematic review of current literature. Ticks Tick Borne Dis 2018;9(06): 1377-1382

44 Parija SC, Kp D, Venugopal H. Diagnosis and management of human babesiosis. Trop Parasitol 2015;5(02):88-93

45 Kletsova EA, Spitzer ED, Fries BC, Marcos LA. Babesiosis in Long Island: review of 62 cases focusing on treatment with azithromycin and atovaquone. Ann Clin Microbiol Antimicrob 2017;16 (01):26 
46 Sigurjonsdottir VK, Feder HM Jr., Wormser GP. Anaplasmosis in pediatric patients: Case report and review. Diagn Microbiol Infect Dis 2017;89(03):230-234

47 Glaser C, Christie L, Bloch KC. Rickettsial and ehrlichial infections. Handb Clin Neurol 2010;96:143-158

48 Ismail N, Bloch KC, McBride JW. Human ehrlichiosis and anaplasmosis. Clin Lab Med 2010;30(01):261-292

49 Stromdahl EY, Randolph MP, O'Brien JJ, Gutierrez AG. Ehrlichia chaffeensis (Rickettsiales: Ehrlichieae) infection in Amblyomma americanum (Acari: Ixodidae) at aberdeen proving ground, Maryland. J Med Entomol 2000;37(03): 349-356

50 Schutze GE, Buckingham SC, Marshall GS, et al; Tick-borne Infections in Children Study (TICS) Group. Human monocytic ehrlichiosis in children. Pediatr Infect Dis J 2007;26(06):475-479

51 Weil AA, Baron EL, Brown CM, Drapkin MS. Clinical findings and diagnosis in human granulocytic anaplasmosis: a case series from Massachusetts. Mayo Clin Proc 2012;87(03):233-239

52 Bakken JS, Dumler JS. Human granulocytic ehrlichiosis. Clin Infect Dis 2000;31(02):554-560 